\title{
Experimental and Theoretical Studies on the Movements of Two Bubbles in an Acoustic Standing Wave Field
}

\author{
Junjie Jiao ${ }^{1,2}$, Yong $\mathrm{He}^{1 *}$, Thomas Leong ${ }^{3}$, Sandra E. Kentish ${ }^{4}$, \\ MuthupandianAshokkumar ${ }^{2 \#}$, Richard Manasseh ${ }^{3}$ and Judy Lee ${ }^{4 *}$ \\ ${ }^{1}$ Department of Mechanical Engineering, Nanjing University of Science and Technology, Jiangsu, China \\ ${ }^{2}$ Particulate Fluids Processing Centre, School of Chemistry, University of Melbourne, Parkville, VIC. \\ 3010 Australia ( ${ }^{\#}$ Adjunct Professor, Chemistry Department, King Abdulaziz University, Jeddah, Saudi \\ Arabia) \\ ${ }^{3}$ Mechanical Engineering, Faculty of Engineering \& Industrial Sciences, Swinburne University of \\ Technology, Hawthorn,VIC, 3122, Australia \\ ${ }^{4}$ Particulate Fluids Processing Centre, Chemical and Biomolecular Engineering, University of Melbourne, \\ Parkville, VIC, 3010 Australia
}

*Corresponding Authors

yhe1964@mail.njust.edu.cn; Tel: +86-25-84315790

jytlee@unimelb.edu.au; Tel: +61-03-9035 8575

\begin{abstract}
When subjected to an ultrasonic standing-wave field, cavitation bubbles smaller than the resonance size migrates to the pressure antinodes. As bubbles approach the antinode, they also move towards each other and either form a cluster or coalesce. In this study the translational trajectory of two bubbles moving toward each other in an ultrasonic standing wave at $22.4 \mathrm{kHz}$ was observed using an imaging system with a high-speed video camera. This allowed the speed of the approaching bubbles to be measured for much closer distances than those reported in the prior literature. The trajectory of two approaching bubbles was modeled using coupled equations of radial and translational motions, showing similar trends with the experimental results. We also indirectly measured the secondary Bjerknes force by monitoring the acceleration when bubbles are close to each other under different acoustic pressure amplitudes. Bubbles begin to accelerate towards each other as the distance between them gets shorter and this acceleration increases with increasing acoustic pressure. The current study provides experimental data that validates the theory on the movement of bubbles and forces acting between them in an acoustic field that will be useful in understanding bubble coalescence in an acoustic field.
\end{abstract}

\section{Key words:}

Bjerknes forces

Acoustic pressure

Two bubble dynamics

Two bubble approach velocity 


\section{Introduction}

The uses of ultrasound are diverse, ranging from the medical field ${ }^{1-4}$ to emulsions ${ }^{5-7}$ and degradation of pollutants ${ }^{8,9}$. In all these applications the operational efficiency is governed by the cavitation bubble population and the size of the cavitation bubbles, which in turn are significantly affected by the coalescence process ${ }^{10-13}$. Before the coalescence process can take place in an acoustic field, the bubbles have to first be drawn towards each other and brought into contact. Therefore it is important to understand the various forces that control the translational movement of bubbles in an acoustic field, namely the acoustic and hydrodynamic forces. The two acoustic forces, identified as the primary and secondary Bjerknes forces, were first reported by Bjerknes in 1906 and $1909^{14,15}$. The primary Bjerknes force depends on the pressure gradient and the average oscillation of a bubble within one cycle, and can force bubbles to move towards the pressure antinodes or pressure nodes, depending on the size of the bubble ${ }^{16-19}$. The secondary Bjerknes force can induce repulsion or attraction between two bubbles, depending upon the average volume oscillation changes and the distance between them ${ }^{18-20}$.

Eller observed that bubbles are trapped near pressure antinodes if the bubble radius is smaller than the resonance size due to the balance between the primary Bjerknes and buoyancy forces ${ }^{21}$. Crum measured the velocity of two bubbles as they move close to each other and modeled the velocity profile of approaching bubbles by considering the primary and secondary Bjerknes forces and the drag forces together ${ }^{22}$. In this earlier study, the effect of bubble oscillation was not taken into account and the data obtained were for large bubble-bubble distances between $2 \mathrm{~mm}$ to $10 \mathrm{~mm}^{22}$. Parlitz et al. ${ }^{19}$ used the particle model, based on Hinsch's research ${ }^{23}$ to study bubble structures in an acoustic field. In this particle model, individual bubbles were treated as moving particles. All forces acting on the bubbles, namely, mass, primary and secondary Bjerknes forces, and drag forces were kept in balance. This model is suitable for simulating bubble structures and it can also be applied to simulate the trajectory of two bubbles in standing and travelling wave fields ${ }^{24}$.

The translational motion of bubbles is related to their oscillations and the pressure around the bubble at a given position. However, the particle model does not take into account coupled 
bubble volume oscillations, resulting in errors in bubble translation when bubbles are subjected to acoustic forces. Watanabe and Kukita performed numerical calculations on a spherical bubble in an acoustic field, and showed that the radial response is affected by the translational motion of the bubble ${ }^{25}$. Doinikov ${ }^{26}$ similarly used the Lagrangian formalism to derive coupled equations of radial and translational motions of a spherical bubble in an acoustic field. Using this model for numerical simulations, he showed that bubbles undergo a swing motion around pressure antinodes when the pressure amplitude is high enough. For two bubbles, Harkin et al. ${ }^{27}$ presented and analyzed a model for the spherical pulsations and translational motions of a pair of interacting gas bubbles in an incompressible liquid. However, their study mainly focused on the theoretical aspects of the direction of the secondary Bjerknes force and the changes brought about by the differences in their initial separation distances and pressure amplitudes. Xi and Cegla ${ }^{28}$ used the coupled translational and radial model developed by Doinikov ${ }^{26}$ to study the bubble movement near a solid surface in a weak standing wave field $(11.5 \mathrm{kPa})$. Their model could explain multi-bubble translations by considering the balance between acoustic and hydrodynamic forces on bubbles in a liquid medium.

As discussed above, when bubbles move in an acoustic field, their movements are controlled by acoustic and hydrodynamic forces simultaneously leading to translational motion of the bubbles. Experimental studies on mutual interaction forces between pulsating bubbles are limited as stated by Doinikov et al. $^{29}$ : "there exists a problem which makes carrying out such experiments difficult. This is that the primary Bjerknes force, as a rule, far exceeds the secondary one. As a result, the separation and measuring of the latter is extremely awkward." Thus, there are no detailed experimental data on the translational motion of two approaching bubbles at close distances less than $2 \mathrm{~mm}$, where secondary Bjerknes force and the effect of acoustic pressure become significant. This study presents experimental results on two bubbles moving towards each other in a standing wave field under acoustic pressure amplitudes between $10 \mathrm{kPa}$ and $40 \mathrm{kPa}$. The experimental results are compared with simulations computed using the theory developed by Doinikov ${ }^{26}$. 


\section{Experimental Method}

A schematic diagram of the bubble observation system, very similar to that described by Ashokkumar and Grieser ${ }^{30}$ is shown in Figure 1(a). A cylindrical Pyrex cell with a diameter of $6.5 \mathrm{~cm}$ and height of $11.5 \mathrm{~cm}$ containing two parallel flat surfaces for viewing and back lighting purposes was used. A hollow cylindrical Lead Zirconate Titanate (PZT) piezo ceramic transducer (American Piezo Ceramics Inc. Z7) was attached to the bottom of the cell. The transducer was driven by a programmable $15 \mathrm{MHz}$ Hameg function generator (HM8131-2) at the transducer's resonance frequency of $22.4 \mathrm{kHz}$ via a Krohn-Hite power amplifier (Model 7500). The bubble images were magnified using a long-distance microscope $(6.3 \times$ magnification, LeicaZ16 APO), and recorded using a high-speed video camera (Y7-PIV, IDT) at a frame rate of 2000 frames per second. The acoustic pressure was measured using an interchangeable $1 \mathrm{~mm}$ PVdF needle hydrophone (Precision Acoustics Ltd1.0 mm needle with preamp). The hydrophone also allowed the antinode to be accurately located. The acoustic pressure used in this study ranged from $10 \mathrm{kPa}$ to $40 \mathrm{kPa}$.

The water was firstly degassed to avoid any cavitation that might affect the translational motion of the two bubbles. A bubble was then injected and trapped at the pressure antinode, followed by the injection of a second bubble. The approach of a second bubble towards the first bubble and the trajectories of the two bubbles were recorded and analyzed frame by frame using Image $\mathbf{J}$ software to allow the bubble position and velocity to be determined.

\section{Mathematical model for bubble translational path}

As mentioned, the translational motion of a bubble in an acoustic field is controlled by both acoustic and hydrodynamic forces. The position of the two bubbles is schematically shown in Figure 1 (a) and the different forces controlling the motion of the two bubbles, mainly along the y axis, are illustrated in Figure 1 (b). Therefore, the focus of this study is the translational motion of two bubbles along the $y$ axis. 
When a bubble is moving in a standing wave field, the primary Bjerknes force $\left(F_{p}\right)$ is related to the bubble position and bubble volume oscillation, which is given by Equation $1{ }^{18}$

$$
F_{p}=-4 / 3 \pi P_{a} k \sin (k d) R^{3}(t) \sin (\omega t)
$$

Where $k$ is the wave number, $R$ is the time-dependent radius, $P_{a}$ is the acoustic pressure amplitude at the pressure antinodes, $t$ is time, $\omega$ is the angular frequency, and $d$ is the distance between the center of the bubble and the nearest pressure anti-node measured in the y-direction.

The secondary Bjerknes force between two bubbles is given by Equation $2^{2}$ :

$$
F_{B}=-\frac{\rho}{4 \pi r_{12}^{2}}\left\langle\dot{V}_{1} \dot{V}_{2}\right\rangle
$$

Where \langle\rangle denotes the time average, $V_{1}$ and $V_{2}$ are the volume of the first and second bubble, $\rho$ is the liquid density, $r_{12}$ is the separation distance between two bubbles, and the over-dot denotes the time derivative.

For hydrodynamic forces in an acoustic field, the drag forces along the $x$ and $y$ axis are given by Equation 3 and $4^{16}$ :

$$
\begin{gathered}
F_{v x}=6 \pi \mu R \dot{x} \frac{\mathrm{e}+r\left(2 \mu+3 \mu^{\prime}\right)}{e+3 r\left(\mu+3 \mu^{\prime}\right)} \\
F_{v y}=6 \pi \mu R \dot{y} \frac{\mathrm{e}+r\left(2 \mu+3 \mu^{\prime}\right)}{e+3 r\left(\mu+3 \mu^{\prime}\right)}
\end{gathered}
$$

Where $e$ is the coefficient of surface viscosity, $r$ is the radius of sphere, $\mu$ is the liquid viscosity, $\mu$ ' is the viscosity inside the sphere, and $y$ is the position of the bubble center on the $y$ axis. When a bubble is moving in an acoustic field the bubble radius $r>e$ and $\mu>>$, this reduces the drag force shown in Equation 3 and 4 to $F_{v x}=4 \pi \mu R \dot{x}$ and $F_{v y}=4 \pi \mu R \dot{y}$, respectively.

The buoyancy force is given by Equation 5: 


$$
F_{\text {buoy }}=\frac{4 \pi}{3} R^{3} \mathrm{~g}\left(\rho-\rho_{\text {gas }}\right)
$$

where $\rho_{\text {gas }}$ is the density of gas inside a bubble and $g$ is the acceleration of gravity.

As discussed above, as the bubble moves in an acoustic field, its position changes, and so does the pressure around it. The distance to other bubbles will also change. These changes can affect the bubble oscillation, which in turn can affect the bubble's translational motion. Therefore both bubble translation and oscillation would mutually influence each other when bubbles move in an acoustic field. Doinikov ${ }^{26}$ used the Lagrangian formalism and coupled equations of radial and translational motions of a gas bubble along the $y$ axis given by

$$
\ddot{y}+\frac{3 \dot{R} \dot{y}}{R}=\frac{3 F_{e y}}{2 \pi \rho R^{3}}
$$

where $F_{e y}$ is the external force along the $y$ axis and includes all forces shown in Figure $\mathbf{1}(\mathrm{b})$.

The time-varying bubble radius can be calculated based on the Keller-Miksis equation ${ }^{31}$ However in a two-bubble environment, the Keller-Miksis equation needs to be expanded to include the influence from the boundary conditions and the neighboring bubble. If the bubble shapes are assumed to remain spherical for all times with radii $R_{1}$ and $R_{2}$, the respective pressure generated by bubble 2 on bubble 1 and vice versa is given by Equation $7^{18}$ :

$$
P=\frac{\rho}{r_{12}}\left(R_{2} \ddot{R}_{2}+2 \dot{R}_{2}^{2}\right)
$$

By incorporating Equation 7 into the Keller-Miksis equation, the oscillation of bubble 1 was obtained: 


$$
\begin{gathered}
\left(1-\frac{\dot{\mathrm{R}}_{1}}{\mathrm{c}}\right) R_{1} \ddot{R}_{1}+\frac{3}{2} \dot{R}_{1}^{2}\left(1-\frac{\dot{R}_{1}}{3 c}\right)=\frac{\dot{y}^{2}}{4}+\left(1+\frac{\dot{\mathrm{R}}_{1}}{\mathrm{c}}\right) \frac{p_{s c}}{\rho}+\frac{R}{\rho c} p_{s c}-\frac{\rho}{r_{12}}\left(R_{2} \ddot{R}_{2}+2 \dot{R}_{2}^{2}\right) \\
p_{s c}=\left(p_{0}+\frac{2 \sigma}{R_{10}}\right)\left(\frac{R_{10}}{R_{1}}\right)^{3 \kappa}-p_{0}-\frac{2 \sigma}{R_{1}}-\frac{4 \mu}{R_{1}} \dot{R}_{1}-P_{e x}
\end{gathered}
$$

where $R_{10}$ is the equilibrium radius bubble $1, p_{0}$ is the hydrostatic pressure, $\sigma$ is the surface tension, $\kappa$ is the polytropic exponent of the gas within the bubble and $P_{e x}$ is the external driving signal that is defined for the standing wave as in Equation 10:

$$
P_{e x}=P_{a} \sin (\omega t) \cos (k d)
$$

Exchanging indices $1 \leftrightarrow 2$, we get the equations for bubble 2 .

Prior to making calculations specific to the present experiments, our numerical solutions to Equations 7-10 were validated against results in the literature ${ }^{25,26,28}$.

\section{Results and discussion}

The experimentally observed data on the translational movements of two bubbles in an acoustic field and the corresponding numerical simulation data are presented below. The values of the physical parameters used in this study are $f=22.4 \mathrm{kHz}, \rho=998 \mathrm{~kg} / \mathrm{m}^{3}, P_{0}=101.3 \mathrm{kPa}, c=$ $1480 \mathrm{~m} / \mathrm{s}, \sigma=0.072 \mathrm{~N} / \mathrm{m}, \gamma=1.4$ and $\mu=0.001 \mathrm{~Pa} / \mathrm{s}$. The acoustic pressure used ranges from $10 \mathrm{kPa}$ to $40 \mathrm{kPa}$.

An example of the translational movements of two bubbles in an ultrasound field is displayed in Figure 2 for a pressure amplitude of $P_{a}=40 \mathrm{kPa}$ using data from a selected experimental run. The dashed line at the base of the photographs marks the position of the pressure antinode. The bubble near the pressure antinode is bubble 2, and its radius is $22 \mu \mathrm{m}$ while the bubble furthest away from the pressure antinode is bubble 1 and its radius is $23 \mu \mathrm{m}$. It should be noted that while the Figures and discussion focus on this one experiment, multiple experiments were conducted and produced similar results (see Supporting Information Available). 
The displacement of the bubbles along the $y$ axis and their velocities were obtained using the images shown in Figure 2 and plotted in Figure 3 and Figure 4, respectively. Initially the distance between the two bubbles is about $1.29 \mathrm{~mm}$, and their positions away from the pressure antinode are $0.238 \mathrm{~mm}$ and $1 \mathrm{~mm}$, respectively. From this point both the acoustic and hydrodynamic forces combined stabilize bubble 2 near the antinode and drives bubble 1 towards the antinode, and thus towards bubble 2. When the bubbles are far away from each other, their relative velocities do not vary significantly (Figure 4). As the distance between them decreases, the relative velocity begins to increase and becomes significant when the bubbles are $0.6 \mathrm{~mm}$ apart. As the distance between them narrows further, the relative velocity increases dramatically. Xi et al. ${ }^{28}$ have shown similar increase in the velocity at short distances for a bubble approaching a surface.

The coupled equations of radial and translational motions of two spherical bubbles, Equations 7-10, were used to simulate this movement of bubbles in an acoustic field and the results are compared with the experimental data in Figure 3 and Figure 4. The simulation results slightly underestimate the position of the two bubbles, but overall the trajectories correlate reasonably well with the experimental data. The relative velocity between the two bubbles shows good agreement with the experimental values (Figure 5).

The simulation results of external forces along the $y$ axis are shown in Figure 5. Initially when the bubbles are far apart, the secondary Bjerknes force is relatively weaker compared to the buoyancy and primary Bjerknes forces. This agrees with what has already been reported in the literature ${ }^{28,29}$. For bubble 2 the buoyancy and primary Bjerknes forces are initially balanced, which explains why the position does not change as much for bubble 1 in the y direction as shown in Figure 3. Conversely, for bubble 1 the primary Bjerknes force is large compared to the buoyancy and secondary Bjerknes forces, and drives bubble 1 towards the antinode. As the bubbles get closer, the primary Bjerknes force decreases for bubble 1 and increases slightly for bubble 2. The secondary Bjerknes force is shown to increase significantly as the bubble-bubble distance gets shorter, causing the bubbles to accelerate towards each other. 
Secondary Bjerknes force has been theoretically ${ }^{32}$ shown to increase with an increase in acoustic pressure. This has been used by Hatanaka et al. ${ }^{13}$ to explain the maximum intensity observed in multibubble sonoluminescence (MBSL) with an increase in acoustic power: an increase in secondary Bjerknes force increases bubble coalescence, which in turn enhances the number of active bubbles and hence MBSL intensity. However too much coalescence at high acoustic powers can cause bubbles to grow too large and be expelled from the antinode, leading to a decrease in the MBSL intensity. Using the same experimental method to obtain the velocity of two bubbles, the effect of increasing acoustic pressures was investigated experimentally. As discussed above, when two bubbles move toward each other, the secondary Bjerknes force increases significantly which causes acceleration of the bubbles towards each other. The acceleration can therefore be used to measure the change in the secondary Bjerknes force under different acoustic pressures (Figure 6). This acceleration is nearly zero when two bubbles are far apart. However, as the distance between the bubbles decreases, a threshold distance is reached at which the relative acceleration between the two bubbles begins to increase very rapidly. The threshold distance at which this acceleration begins to increase, increases with increasing acoustic pressure, from $0.2 \mathrm{~mm}$ at $10 \mathrm{kPa}$ to $0.5 \mathrm{~mm}$ at $40 \mathrm{kPa}$. It can also be seen in Figure 6 that the acceleration is greater at higher acoustic pressures. According to Equation 2, the secondary Bjerknes force is related to the two bubbles' average volume change in one cycle, and to the distance between two bubbles. As the acoustic pressure increases, the bubble oscillation will also become larger and the bubbles will influence each other at a further distance apart. Both an increase in bubble oscillation amplitude and decrease in distance between the two bubbles lead to an increase in the secondary Bjerknes force. This eventually results in two bubbles coming into contact with each other, to either form a cluster, coalesce, or re-bound.

\section{Conclusions}

The experimental and simulation results presented in this study provide valuable information on the behavior of two bubbles in an acoustic field. When one bubble approached another bubble, a threshold distance was reached at which the secondary Bjerknes force became dominant. With a further decrease in separation distance, the Bjerknes forces increase, leading to the acceleration of the two bubbles towards each other. Using coupled equations of radial and translational 
motions to simulate the trajectory of the bubbles, a good agreement with the experimental data was obtained. The acceleration between two bubbles is higher at higher acoustic pressures and from this data we can estimate the changes in the secondary Bjerknes force. The results presented in this study are useful in the understanding of bubble coalescence in aqueous solutions in an acoustic field, which is an important practical issue in many applications that include ultrasonic degassing and ultrasonic processing of liquids.

\section{Acknowledgements}

We are grateful to Prof Yos Morsi for facilitating the use of the high-speed camera. The financial support through the Australian Research Council for the DECRA (Discovery Early Career Research Award, DE120101567) is also gratefully acknowledged.

\section{Supporting Information Available}

A figure demonstrating the reproducibility of multiple experiments conducted in this study. This information is available free of charge via the Internet at http;//pubs.acs.org 


\section{References}

1. Postema, M.; Mleczko, M.; Schmitz, G. Mutual attraction of oscillating microbubbles Adv. Med. Eng. 2007, 114, 75-80.

2. Zhang, Y.; Guo, X.; Zhang, D.; Gong, X. 20th International Congress on Acoustics, ICA2010, Sydney, Australia, 2010, pp 716.

3. Dayton, P. A.; Morgan, K. E.; Klibanov, A. L.; Brandenburger, G.; Nightingale, K. R.; Ferrara, K. W. A Preliminary Evaluation of The Effects of Primary and Secondary Radiation Forces on Acoustic Contrast Agents IEEE Trans. Ultrason. Ferroelectr. Freq. Control 1997, 44, 1264-1277.

4. Garbin, V.; Overvelde, M.; Dollet, B.; de Jong, N.; Lohse, D.; Versluis, M. Unbinding of Targeted Ultrasound Contrast Agent Microbubbles by Secondary Acoustic Forces Phys. Med. Biol. 2011, 56, 6161-6177.

5. Biggs, S. R.; Grieser, F. Preparation of Polystyrene Latex with Ultrasonic Initiation Macromolecules 1995, 28, 4877-4882.

6. Bradley, M.; Ashokkumar, M.; Grieser, F. Sonochemical Production of Fluorescent and Phosphorescent Latex Particles J. Am. Chem. Soc. 2003, 125, 525-529.

7. Leong, T. S. H.; Wooster, T. J.; Kentish, S. E.; Ashokkumar, M. Minimising Oil Droplet Size using Ultrasonic Emulsification Ultrason. Sonochem. 2009, 16, 721-727.

8. Yim, B.; Nagata, Y.; Maeda, Y. Sonolytic Degradation of Phthalic Acid Esters in Aqueous Solutions. Acceleration of Hydrolysis by Sonochemical Action J. Phys, Chem. A 2002, 106, 101-107.

9. Vinodgopal, K.; Ashokkumar, M.; Grieser, F. Sonochemical Degradation of a Polydisperse Nonylphenol Ethoxylate in Aqueous Solution J. Phys, Chem. B 2001, 105, 3338-3342.

10. Ashokkumar, M.; Hall, R.; Mulvaney, P.; Grieser, F. Sonoluminescence from Aqueous Alcohol and Surfactant Solutions J. Phys. Chem. B. 1997, 101, 10845-10850.

11. Lee, J.; Kentish, S. E.; Muthupandian, A. The Effect of Surface-Active Solutes on Bubble Coalescence in the Presence of Ultrasound J. Phys, Chem. B 2005, 109, 5095-5099.

12. Lee, J.; Ashokkumar, M.; Kentish, S. E.; Grieser, F. Determination of the Size Distribution of Sonoluminescence Bubbles in a Pulsed Acoustic Field J. Am. Chem. Soc. 2005, 127, 16810-16811.

13. Hatanaka, S.; Yasui, K.; Tuziuti, T.; Kozuka, T.; Mitome, H. Quenching Mechanism of Multibubble Sonoluminescence at Excessive Sound Pressure Jpn. J. Appl. Phys. 2001, 40, 3856-38620.

14. Bjerknes, V. Fields of Force; The Columbia University Press: New York, 1906.

15. Bjerknes, V. Die Krajlfelder (Vieweg, Braunschweig) 1909.

16. Haberman, W. L.; Morton, R. K. In David Taylor Model Basin Report 802, 1953.

17. Boussinesq, J. Vitesse de la chute lente, devenue uniform, d'unegoutteliquide pheirique, dans un fluidevisqueux de poidsspecifiquemoindre Ann. de Chimie et de Phys. 1918.

18. Mettin, R.; Akhatov, I.; Parlitz, U.; Ohl, C. D.; Lauterborn, W. Bjerknes Forces Between Small Cavitation Bubbles in a Strong Acoustic Field Phys. Rev. E 1997, 56, 2924-2931.

19. Parlitz, U.; Mettin, R.; Luther, S.; Akhatov, I.; Voss, M.; Lauterborn, W. Spatio-Temporal Dynamics of Acoustic Cavitation Bubble Clouds Phil. Trans. R. Soc. Lond. A 1999, 357, 313-334.

20. Oguz, H. N.; Prosperetti, A. A Generalization of the Impulse and Virial Theorems with an Application to Bubble Oscillations J. Fluid. Mech. 1990, 1990, 143-162.

21. Eller, A. Force on a Bubble in a Standing Acoustic Wave J. Acoust. Soc. Am. 1968, 43, 170-171.

22. Crum, L. A. Bjerknes Forces on Bubbles in a Stationary Sound Field J. Acoust. Soc. Am. 1975, 57, 1363-1370.

23. Hinsch, K. Proc. 6th Int. Symp. on Nonlinear Acoustics, Moscow, 1975, pp 26-34.

24. Koch, P.; Krefting, D.; Tervo, T.; Mettin, R.; Lauterborn, W. In Proc. of the 18th Int. congress on Acoustics ICA 2004: Kyoto, Japan, 2004, p V-3571.

25. Watanabe, T.; Kukita, Y. Translational and Radial Motions of a Bubble in an Acoustic Standing Wave Field Phys. Fluids A 1993, 5, 2682-2687.

26. Doinikov, A. A. Translational Motion of a Spherical Bubble in an Acoustic Standing Wave of High Intensity Phys. Fluids A 2002, 14, 1420-1425.

27. Harkin, A.; Kaper, T. J.; Nadim, A. Coupled Pulsation and Translation of Two gas Bubbles in a Liquid J. Fluid. Mech. 2001, 445, 377-411. 
28. Xi, X.; Cegla, F.; Mettin, R.; Holsteyns, F.; Lippert, A. Collective Bubble Dynamics Near a Surface in a Weak Acoustic Standing Wave Field J. Acoust. Soc. Am. 2012, 132, 37-48.

29. Doinikov, A. A.; Zavtrak, S. T. On the Mutual Interaction of Two Gas Bubbles in a Sound Field Phys. Fluids 1995, 7, 1923-1930.

30. Ashokkumar, M.; Grieser, F. Single-Bubble Sonophotoluminescence J. Am. Chem. Soc. 2000, 122, 1200112002 .

31. Keller, J. B.; Miksis, M. Bubble Oscillations of ILarge Amplitude J. Acoust. Soc. Am. 1980, 68, 628-633.

32. Lauterborn, W.; Kurz, T.; Mettin, R.; Ohl, C. D. Experimental and Theoretical Bubble Dynamics Adv. Chem. Phys. 1999, 110, 295-380. 


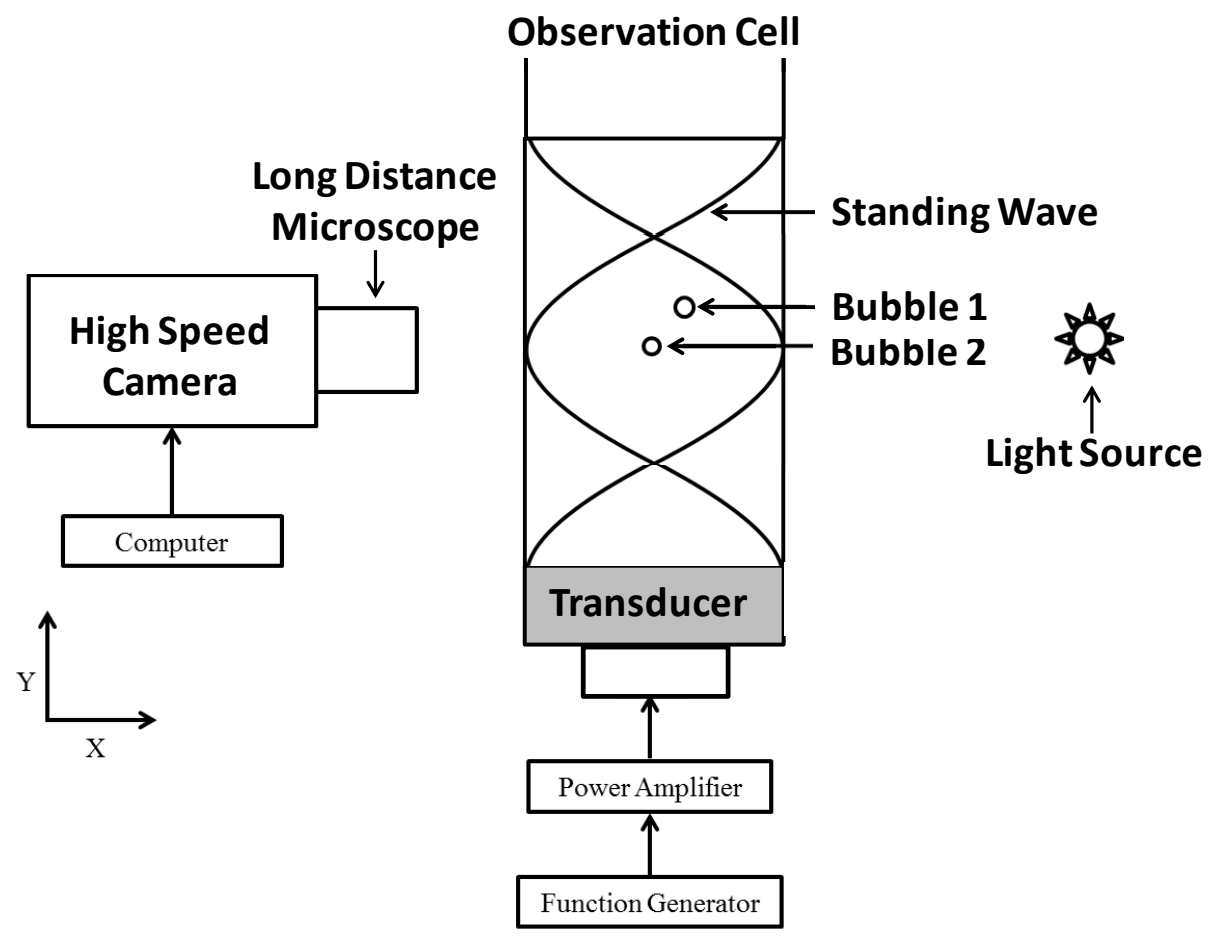

(a)

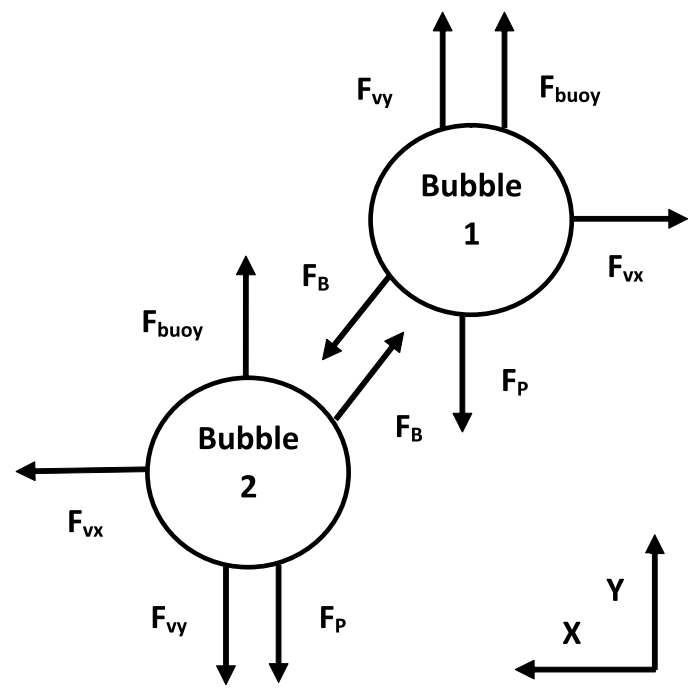

(b)

Figure 1: (a) Imaging system for observing the bubble behavior and the position of two bubbles in a standing wave within the observation cell and (b) The relationship between different external forces on bubble 1 and bubble 2 . 


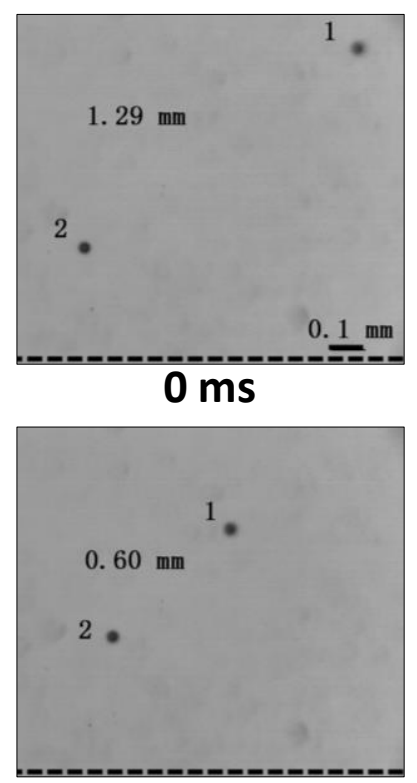

$40 \mathrm{~ms}$

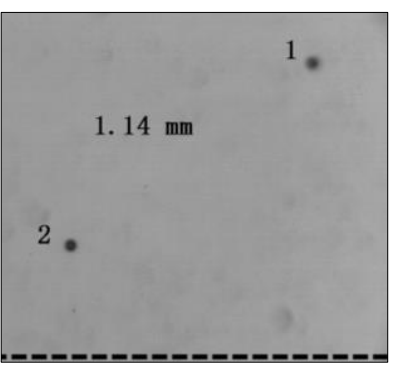

$10 \mathrm{~ms}$

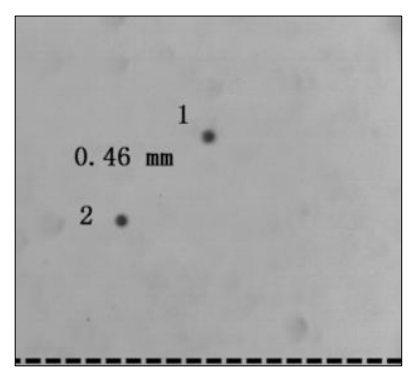

$45 \mathrm{~ms}$

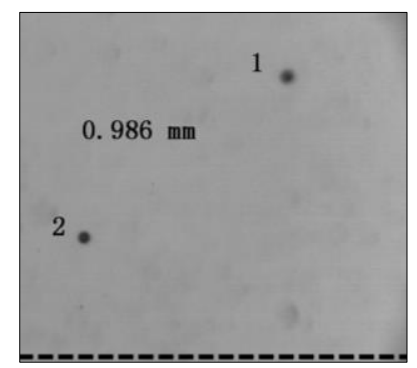

$20 \mathrm{~ms}$

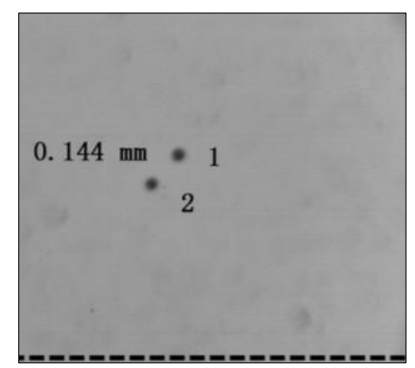

$50 \mathrm{~ms}$
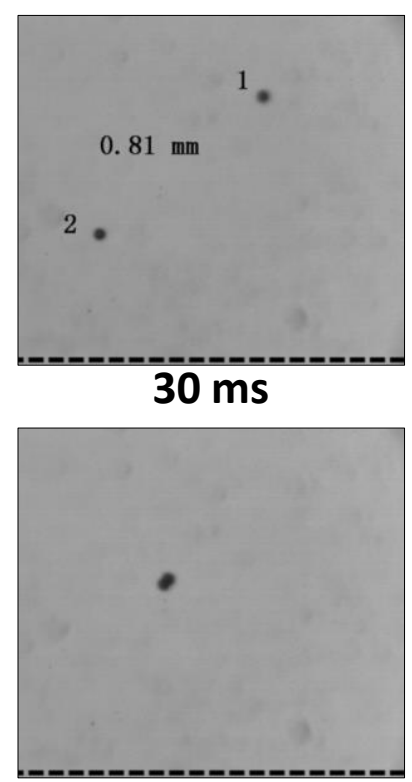

$50.5 \mathrm{~ms}$

Figure 2: Selected frames showing the translations of two bubbles. The distance between the centers of the two bubbles are denoted in the photographs. The dash line at the bottom indicates the position of the pressure antinode. 


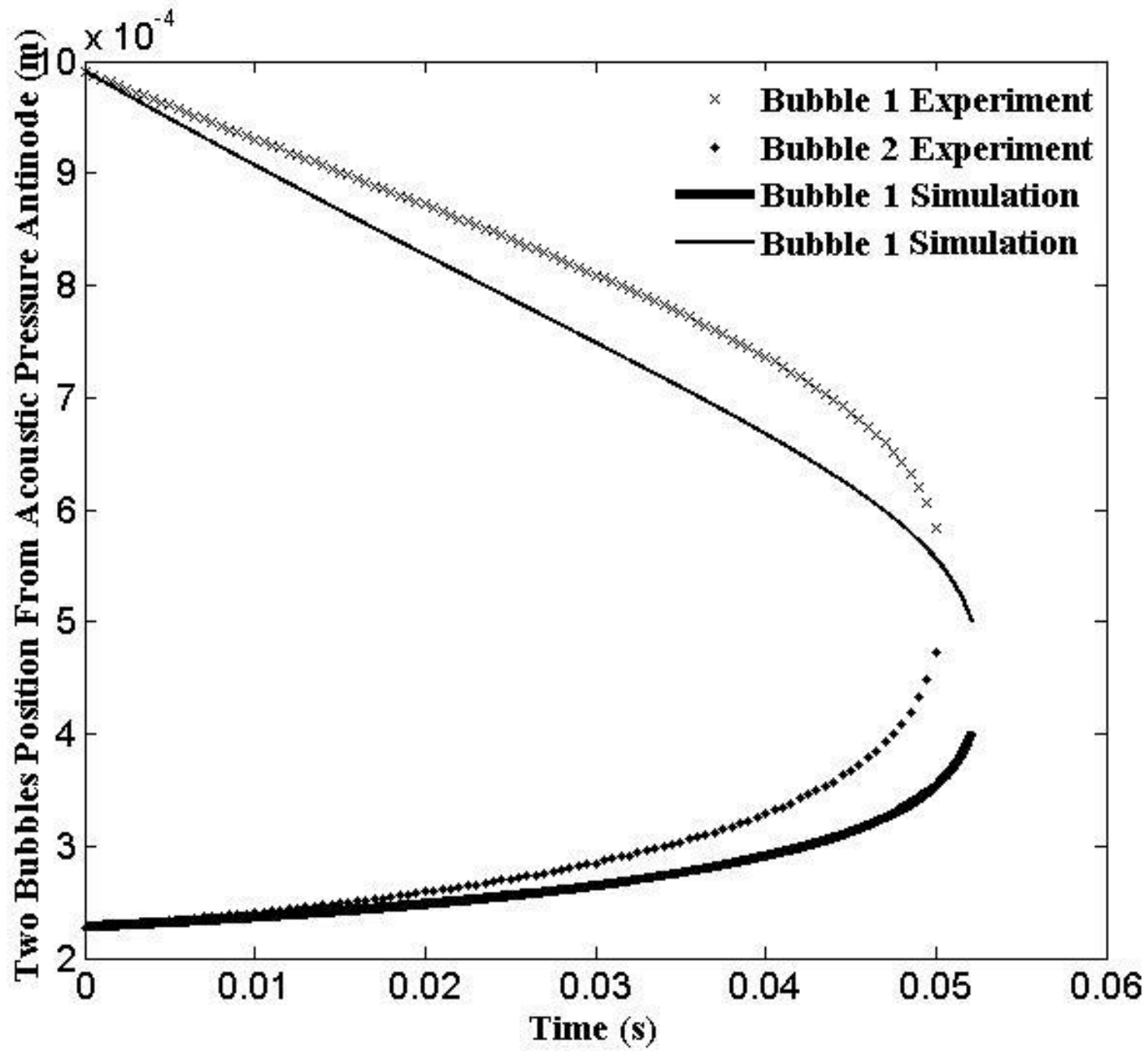

Figure 3: Positions of the bubbles as a function of time at an acoustic pressure of $40 \mathrm{kPa}$. Bubble 1 and bubble 2 radii are $23 \mu \mathrm{m}$ and $22 \mu \mathrm{m}$, respectively. 


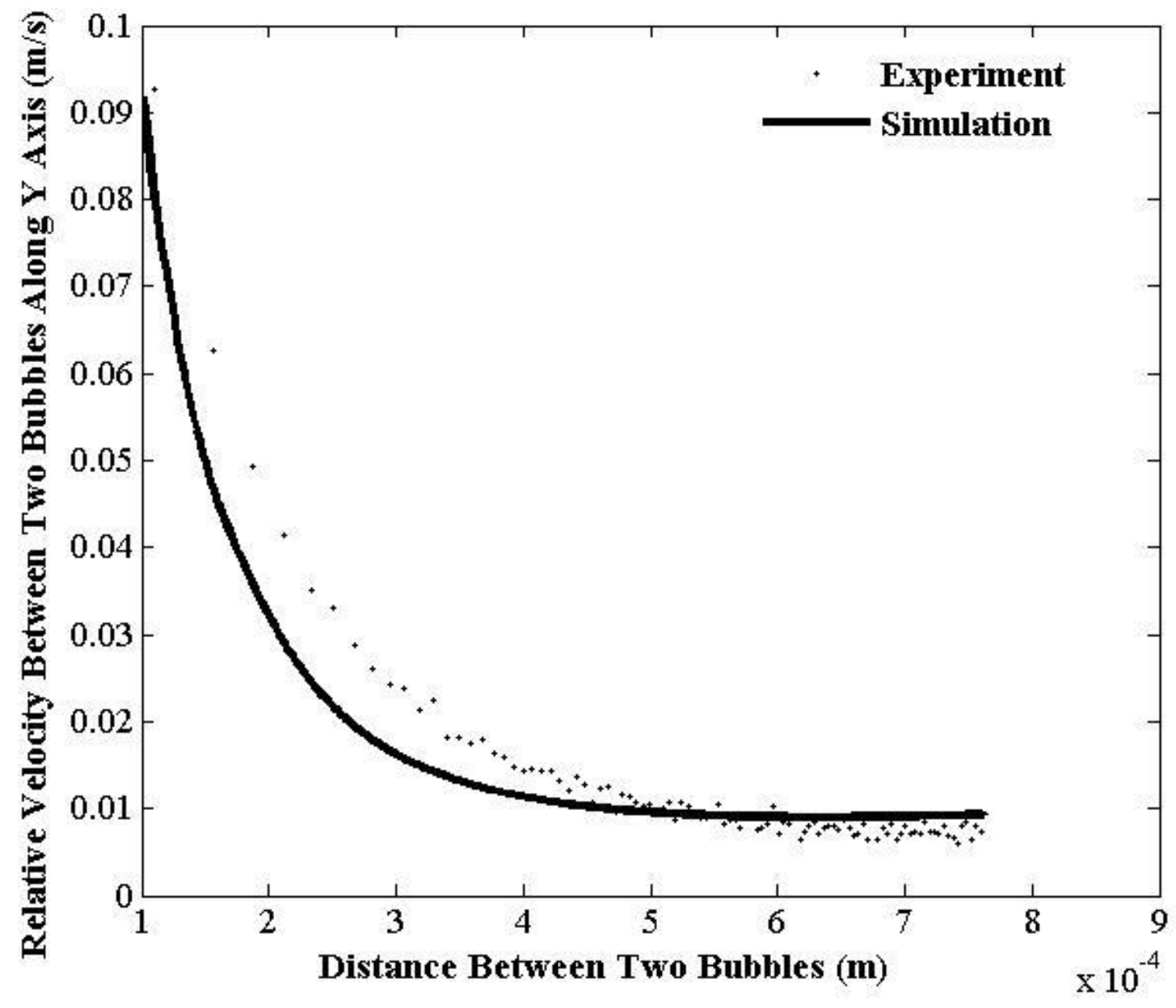

Figure 4: Velocity of the bubbles as a function of distance between two bubbles for an acoustic pressure of $40 \mathrm{kPa}$. Bubble 1 and bubble 2 radii are $23 \mu \mathrm{m}$ and $22 \mu \mathrm{m}$, respectively. 


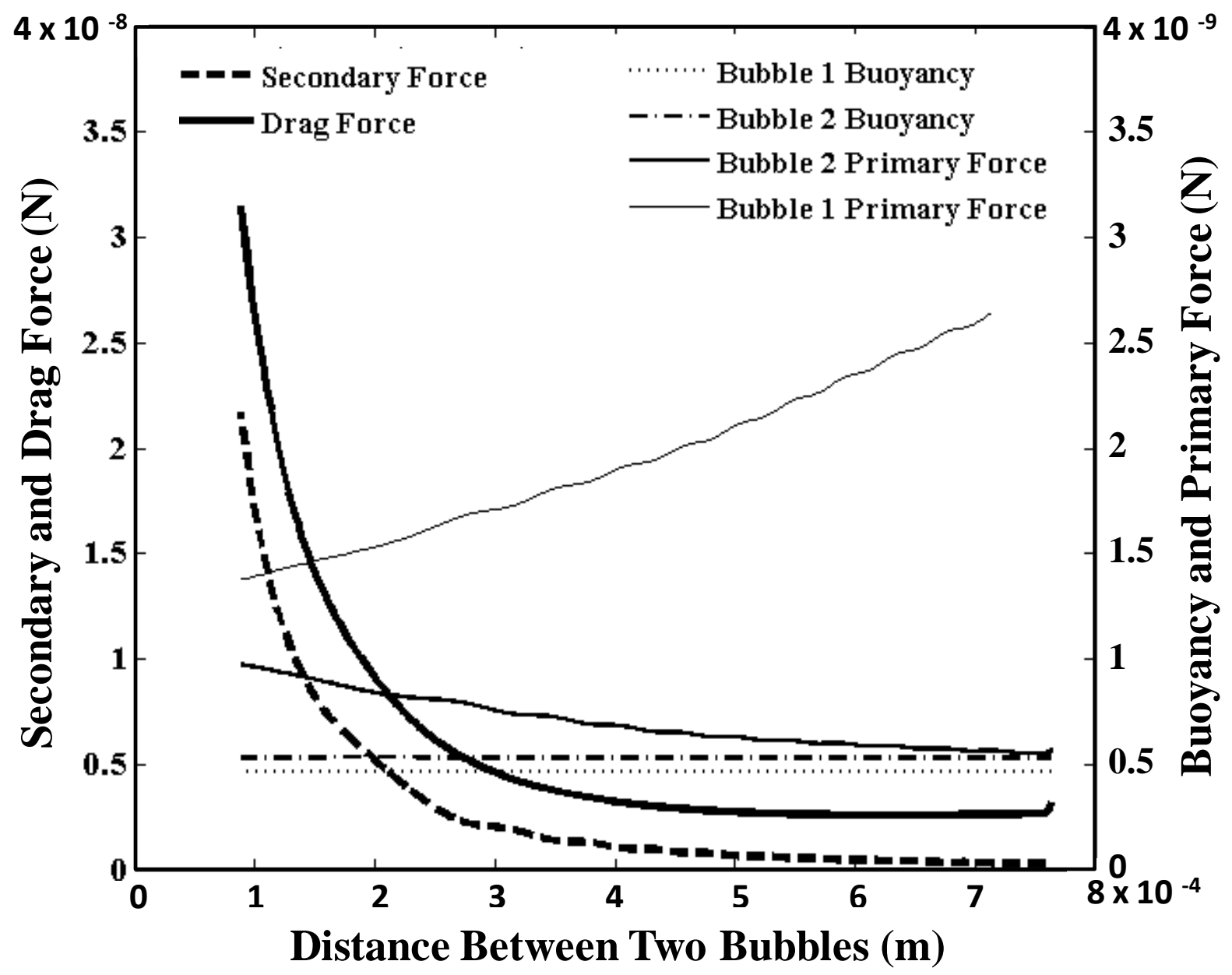

Figure 5: The change of secondary Bjerknes and drag force (right axis) and primary Bjerknes force and buoyancy force (left axis) as a function of distance for an acoustic pressure of $40 \mathrm{kPa}$. Bubble 1 and bubble 2 radii are $23 \mu \mathrm{m}$ and $22 \mu \mathrm{m}$, respectively. 


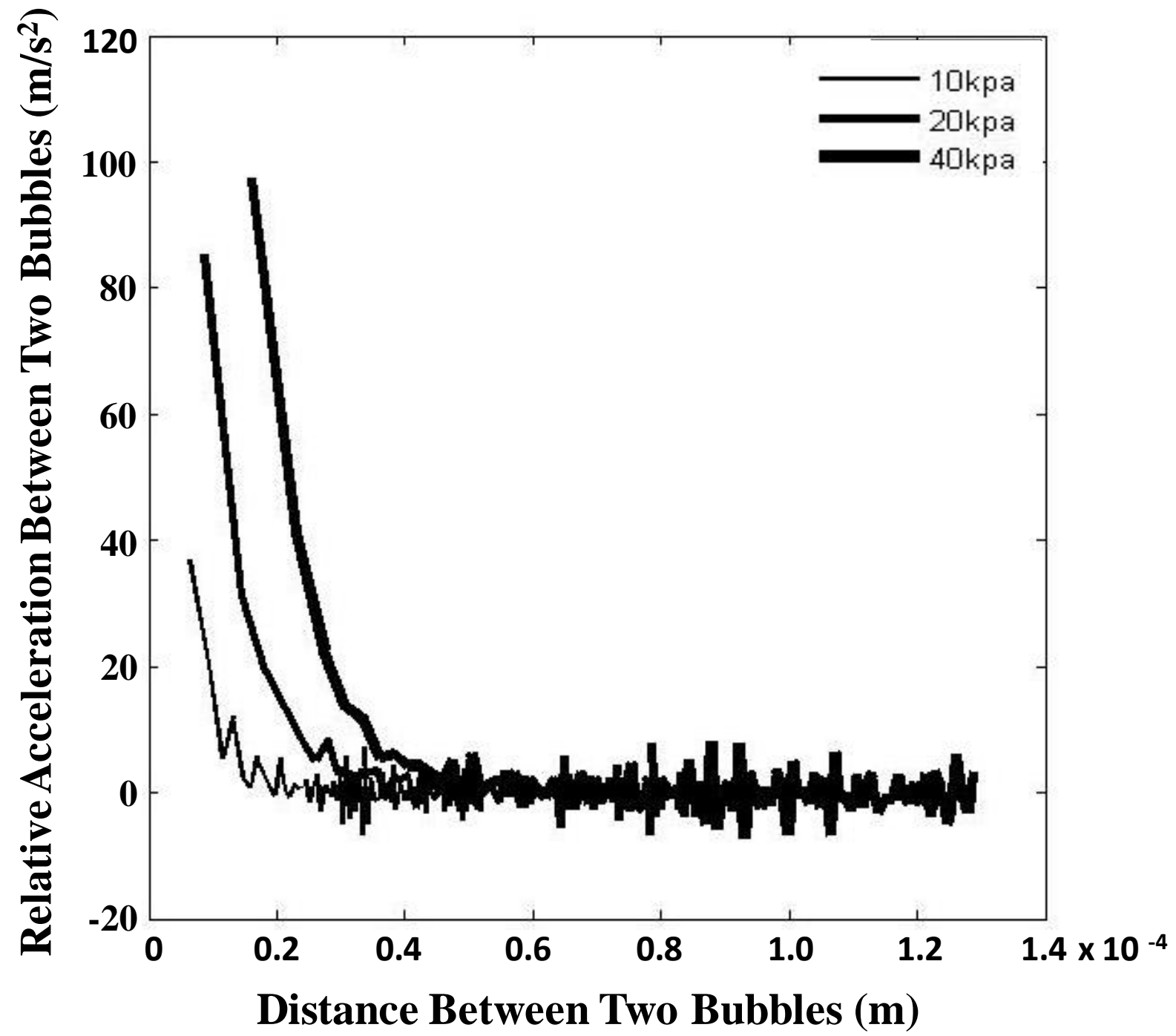

Figure 6: Relative acceleration of two bubbles in different acoustic pressure fields as a function of time. Bubble sizes were $20 \mu \mathrm{m}$ and $19 \mu \mathrm{m}$ for $10 \mathrm{kPa}, 24 \mu \mathrm{m}$ and $23 \mu \mathrm{m}$ for $20 \mathrm{kPa}$, and $23 \mu \mathrm{m}$ and $22 \mu \mathrm{m}$ for $40 \mathrm{kPa}$. 